\title{
A Multi-objective Optimization Evaluation Based on Experimental Datum
}

\author{
Lei Wang \\ School of Mechanical Engineering \& Automation \\ Northeastern University \\ Shenyang, China
}

\author{
Tianzhong Sui* \\ School of Mechanical Engineering \& Automation \\ Northeastern University \\ Shenyang, China \\ *Corresponding author
}

\begin{abstract}
Aiming at multi-objective optimization problem of black-box system, the model of multi-objective optimization EDANN-NGA-FCE (Experimental Design-Artificial Neural Networks-Niche Genetic Algorithm-Fuzzy Comprehensive Evaluation) is established. A fuzzy evaluation for multi-objective Pareto-optimal set is main work in this paper. With the nearness, Pareto-optimal set are evaluated as an objective method. And with expert estimation, Pareto-optimal set are evaluated as a subjective method. On the basis this, combining the two methods, a fuzzy comprehensive evaluation result can be obtained. A subjective and objective standard can be taken into consideration using this method. And the contradiction results and the problem of difficult evaluation can be solved efficiently.
\end{abstract}

Keywords-fuzzy comprehensive evaluation; nearness; black-box; multi-objective optimization

\section{INTRODUCTION}

The multi-objective optimization problem has always been a tricky issue. Parameter optimization problems in engineering practice are mostly multi-objective parameter optimization problem.

Early in multi-objective optimization study, the weighted method was used to synthesize multiple objectives into a single objective. The optimized results were single and did not guarantee optimality. Gradually in the development of evolutionary algorithms such as Pareto genetic algorithm [1], using the concept of Pareto optimality, combined with the hierarchical ordering [2] and the niche technique [3] and other methods to obtain uniform distribution non inferior solution set of the solution space, feasibility research mainly focus on improving the efficiency of algorithm, the method feasibility and so on.

Appling fuzzy theory, fuzzy nearness is used as an objective evaluation method for multi-objective Pareto solution in this paper. The expert scoring is used as the subjective evaluation method of multi-objective Pareto solution. On the basis, combining the two methods, results are obtained based on the fuzzy comprehensive evaluation. This method gives consideration to both the subjective and objective standard, which can effectively solve the multi-objective optimization problem that each target is contradictory, difficult to evaluate.

\section{Solving Scheme ABout Black Box Multi- OBJECTIVE OPTIMIZATION PROBLEM}

According to the black box objective optimization problem, because internal and mutual relationship is not clear, and we can't get the system specific mathematical model expression. In this case, we should often use test design method, through a lot of experiments and data analysis for the system parameters, even so, the obtained parameters are not always the best results.

The author developed a black box multi-objective optimization and evaluation system model ED - ANN - NGA FCE (experimental design / artificial neural network / niche genetic algorithm / fuzzy comprehensive evaluation), to solve the black box system multi-objective optimization problem. System model is shown as Figure I. The system includes four basic processes. Each process using different technical solutions, and they contact each other, forming a complete system.

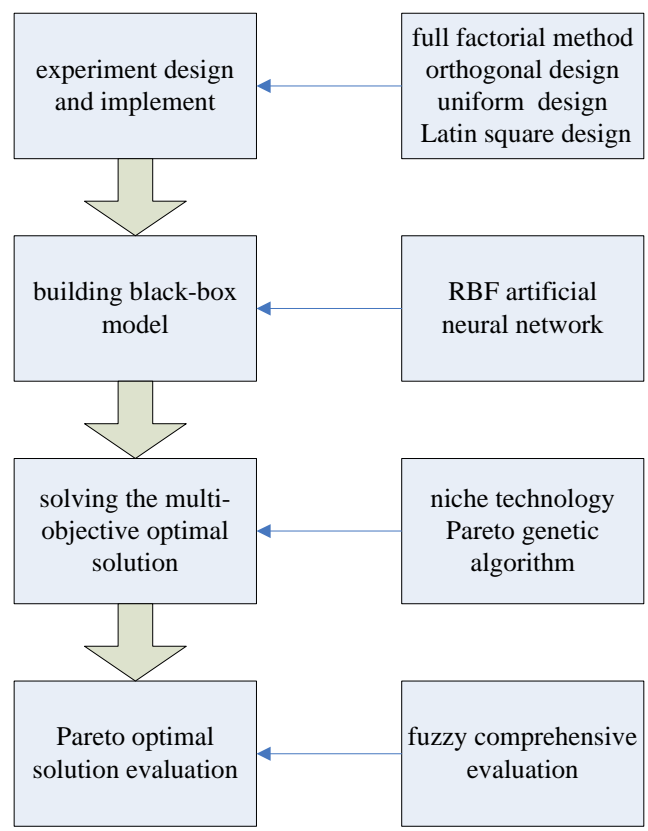

FIGURE I SYSTEM MODEL OF BLACK BOX MULTI-OBJECTIVE OPTIMIZATION AND EVALUATION

In experimental design and implementation process, the full factorial method, orthogonal design, uniform design, Latin 
square design [1] is based on experiments in order to obtain the experimental sample data, and prepare for the establishment of a black box model. Full factorial method in experimental design considering all the different combinations of all levels in all experimental factors; orthogonal design method using orthogonal table to a reasonable arrangement test, so only do fewer number of tests to determine the better condition. Its characteristic is: different level of each factor in the same number of test; any two factors of the various levels of the match, in the test and the same number of occurrences. Uniform with "neat comparable; uniform design method in the application of the principle of multi-dimensional numerical integration based on number theory, constructed a set of uniform design table to uniform design. Uniform design only considers the design of a test pilot evenly spread in the test range.

In constructing the black box model, the acquired experimental data of the input and output are as artificial neural network's input and output, establishing a nonlinear mapping relationship of factors and target. RBF network structure is similar to a multilayer forward network. It is a kind of three layer forward network. The first layer is the input layer consisting by the source node; second layer to the hidden layer and the needs of the number of hidden units, as described, the transformation function of the hidden units is on RBF. It is the center of symmetry radial symmetry and attenuation nonlinear function; third layer is the output layer, and the role of the input mode to respond. The mapping of input to output is non-linear, and the hidden layer space to the output space mapping is linear, which can greatly accelerate the learning speed and avoid local minimum problem.

There is fundamentally different between Multi-objective optimization problem and single-objective optimization problems. Multi-objective optimization solution is not unique and exist a set of optimal solutions. The best way to solve multi-objective optimization problem is to be evenly distributed Pareto optimal solution set, according to the different requirements and wishes, from which to select the most satisfactory results. During multi-objective optimization, Pareto genetic algorithm is as the optimization solver. At same time, the niche technology is used in the paper. Niche technology is the generation of individuals divided into several categories, each class selected larger individuals of a number of fitness as an excellent representative of a class composed of a group in the population, as well as between different populations, hybridization, variation and produce a new generation of individual groups. Adopting the pre-selection and exclusion mechanisms or sharing mechanism to complete the task. Based on niche genetic algorithm it can better maintain the diversity of solutions and has a high ability of global optimization and convergence speed, particularly suited to complex multimodal function optimization problems. Thus available to the given parameter interval Pareto optimal solution set.

After obtaining Pareto optimal solution set, how to assess them and determine the "optimal" solution? This paper offers two evaluation methods: fuzzy approach degree and fuzzy comprehensive assessment method. Fuzzy nearness is an objective evaluation method. The fuzzy comprehensive evaluation method is a subjective assessment method. This article using the fuzzy comprehensive evaluation methods, while adding the objective and subjective evaluation factor set, which makes the evaluation more comprehensive and accurate.

\section{AN APPLICATION CASE}

Based on the direct optimization method above mentioned, the CAD model shown as Figure II is optimized for multiobjective parameters. The instance is a crossing pipe. It has 6 design factors and 3 design objectives. The purpose is obtaining the dimension parameters which have the shortest length of two pipes' curves and the shortest distance between the two pipes.

The meanings of each design factor and their value ranges are as follows: design variable1 expresses the distance between the datum point V_PNT and left end of the horizontal pipe; design variable2 expresses the distance between the datum point V_PNT and lower end of the horrent pipe; design variable 3 expresses the distance between the datum point V_PNT and the datum plane; design variable4 expresses the distance between the datum point H_PNT and left end of the horizontal pipe; design variable5 expresses the distance between the datum point H_PNT and lower end of horrent pipe, and design variable6 expresses the distance between the datum point H_PNT and the datum plane. The ranges of these variables are (47 77), (51 81), (65 95), (43 73), (59 89), (25 55) respectively.

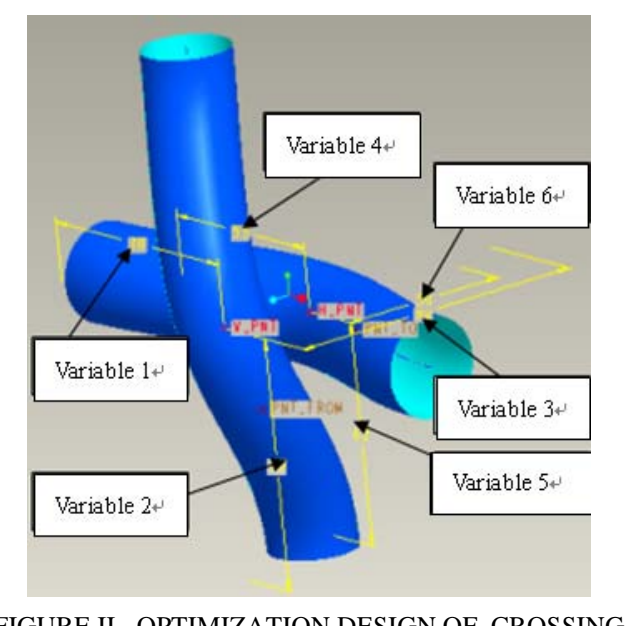

FIGURE II OPTIMIZATION DESIGN OF CROSSING

The meaning about each design objective is as follows: design objective 1 expresses the curve length of the horizontal pipe; design objective2 expresses the curve length of horrent pipe and design objective3 expresses the distance between the two pipes.

Table I is a group of Pareto solutions. Validated by Pro/E, the other solutions are reasonable Pareto solutions except the 1st, 2nd and 7th solutions which don't create proper models. 


\section{A GROUP OF PARETO SOLUTIONS}

TABLE I A GROUP OF PARETO SOLUTIONS

\begin{tabular}{|c|c|c|c|c|}
\hline No. & Var.1 & Var.2 & Var.3 & Var.4 \\
\hline 1 & 51.1670 & 58.9067 & 77.6400 & 61.4774 \\
\hline 2 & 52.1711 & 51.2637 & 79.1776 & 68.7470 \\
\hline 3 & 52.2996 & 51.5649 & 78.5024 & 59.7895 \\
\hline 4 & 52.2996 & 74.8212 & 79.1403 & 45.0200 \\
\hline 5 & 52.6948 & 51.2637 & 78.4900 & 44.7950 \\
\hline 6 & 52.2997 & 74.8212 & 79.1403 & 59.7895 \\
\hline 7 & 47.0048 & 71.9685 & 75.0000 & 71.2994 \\
\hline 8 & 52.9297 & 53.3842 & 75.0353 & 44.9885 \\
\hline 9 & 52.8946 & 80.5359 & 75.0261 & 44.8604 \\
\hline 10 & 52.9352 & 53.3848 & 75.0013 & 44.8897 \\
\hline No. & \multicolumn{2}{|c|}{ Tgt.1 } & Tgt.2 & Tgt.3 \\
\hline 1 & \multicolumn{2}{|c|}{143.0449} & 128.3641 & 1.6200 \\
\hline 2 & \multicolumn{2}{|c|}{145.4009} & 128.1812 & 3.5722 \\
\hline 3 & \multicolumn{2}{|c|}{145.3160} & 135.6608 & 1.3017 \\
\hline 4 & \multicolumn{2}{|c|}{144.5250} & 134.6246 & 3.6187 \\
\hline 5 & \multicolumn{2}{|c|}{144.3106} & 138.8159 & 2.2322 \\
\hline 6 & \multicolumn{2}{|c|}{144.3625} & 134.3831 & 2.0924 \\
\hline 7 & \multicolumn{2}{|c|}{141.8581} & 124.6145 & 2.5583 \\
\hline 8 & \multicolumn{2}{|c|}{143.8294} & 131.2468 & 1.4526 \\
\hline 9 & \multicolumn{2}{|c|}{143.1886} & 130.9115 & 2.5242 \\
\hline 10 & \multicolumn{2}{|c|}{143.8217} & 131.2452 & 2.4517 \\
\hline
\end{tabular}

For this example, use the fuzzy nearness method as follows:

The ideal solution for three target optimization is $F^{*}=[100,100,1]^{T}$; From Table I, seven sets of efficient solutions are:

$$
\begin{aligned}
& F_{1}=[145.3160,135.6608,1.3017]^{T} \\
& F_{2}=[144.5250,134.6246,3.6187]^{T} \\
& F_{3}=[144.3106,138.8159,2.2322]^{T} \\
& F_{4}=[144.3625,134.3831,2.0924]^{T} \\
& F_{5}=[143.8294,131.2468,1.4526]^{T} \\
& F_{6}=[143.1886,130.9115,2.5242]^{T} \\
& F_{7}=[143.8217,131.2452,2.4517]^{T}
\end{aligned}
$$

The Close degree are calculated as follows:

$$
\begin{array}{lllllll}
0.5404 & 0.2416 & 0.3354 & 0.3936 & 0.5585 & 0.3464 & 0.3513
\end{array}
$$

It can be seen that the first group is close to the fifth group, indicating that these two effective solutions can be the optimal solution, which is also consistent with the intuitive selection and artificial.

\section{A. Establishing Evaluation Factors Set}

Three evaluation factors are setting here: manufacturing / procurement costs, construction / maintenance convenience, compact structure, which can establish the evaluation factors, set $\mathrm{U}=$ \{manufacturing / procurement costs, construction / maintenance convenience, compactness $\}$

\section{B. Establishing Comprehensive Evaluation of the Alternative Set}

For this problem, you can set the comment set $\mathrm{V}=\{\mathrm{bad}$, poor, medium, good, excellent $\}$, the corresponding figures expressed as $\mathrm{V}=\{1,2,3,4,5\}$.

\section{The Single Factor Evaluation}

Respectively from the each factor of setting, and determine the evaluation of the alternative set of membership degree of each evaluation factor. With 10 experts scoring, then

(Comprehensive evaluation of the optimal solution alternative set) to (Manufacturing/procurement costs) groups effective solutions expert scoring results are as follows:

$$
\begin{aligned}
& R_{1}^{1}=(0.1,0.5,0.2,0.1,0.1) \quad R_{1}^{2}=(0,0.3,0.4,0.2,0.1) \\
& R_{1}^{3}=(0,0.1,0.5,0.3,0.1) \quad R_{1}^{4}=(0,0,0.3,0.6,0.1) \\
& \underset{\sim}{R_{1}^{5}}=(0,0.1,0.5,0.3,0.1) \underset{\sim}{\sim_{1}^{*}}=(0,0,0.4,0.5,0.1), \\
& R_{1}^{7}=(0,0,0,0.6,0.4)
\end{aligned}
$$

(Comprehensive evaluation of the optimal solution alternative set) to (Construction/maintenance convenience)' membership degree depends on the results of three targets. The pipeline curve is too long or too short, too small or too large are not conducive to the construction and maintenance of pipeline distance, each group effective solutions that experts score is:

$$
\begin{aligned}
& R_{\sim}^{1}=(0 \cdot 1,0 \cdot 7,0 \cdot 1,0 \cdot 1,0) \quad R_{\sim}^{2}=(0,0 \cdot 1,0 \cdot 6,0 \cdot 2,0.1) \\
& R_{2}^{3}=(0,0.2,0.4,0.3,0.1) \quad \stackrel{\sim}{R_{2}^{4}}=(0,0.1,0.2,0.6,0.1) \\
& \underset{\sim}{R_{2}^{5}}=(0,0,0.6,0.4,0){ }_{\sim} R_{2}^{6}=(0,0,0.3,0.5,0.2), \\
& R_{2}^{7}=(0,0,0.1,0.7,0.2)
\end{aligned}
$$

(Alternative set comprehensive evaluation of the optimal solution) to (compactness) membership can be solved by the expert scoring, and we can direct reference to the previously calculated approach degree considerations and confirmed by experts, because of the close degree actually reflects the size of the structure is compact or not. So we can order according to the close degree of efficient solutions. Evaluation set are described as:

$$
\begin{aligned}
& R_{3}^{1}=(0,0,0,0.54,0.54) \quad R_{3}^{2}=(0.24,0.24,0,0,0) \\
& R_{\sim}^{3}=(0,0.34,0.34,0,0){ }_{\sim}^{\prime} R_{3}^{4}=(0,0,0.39,0.39,0) \\
& R_{3}^{5}=(0,0,0,0.56,0.56) \quad R_{\sim}^{6}=(0,0.35,0.35,0,0) \\
& R_{3}^{7}=(0,0.35,0.35,0,0)
\end{aligned}
$$




\section{Establishing Weights Set}

In the three evaluation factors, the weights are determined according to their importance for optimized solution comprehensive evaluation. It can be drawn through the Delphi method or expert score. In these three factors, construction/maintenance convenience is the most important, then compactness, manufacturing/procurement cost is lighter, the weight set can be determined for:

$$
W=(0.2,0.5,0.3)
$$

\section{E. Fuzzy Comprehensive Evaluation:}

Using fuzzy weight vector $\mathrm{W}$ to make $\mathrm{R}$ different rows comprehensive, membership degree can be evaluated as a whole things for each grade of fuzzy subsets. That is fuzzy comprehensive evaluation vector $\mathrm{B}$ :

$$
\begin{gathered}
B_{1}=(0.070,0.450,0.090,0.232,0.182) \\
B_{2}=(0.072,0.182,0.380,0.140,0.070) \\
B_{3}=(0,0.222,0.402,0.210,0.070) \\
B_{4}=(0,0.050,0.277,0.537,0.070) \\
B_{5}=(0,0.020,0.400,0.428,0.188) \\
B_{6}=(0,0.105,0.335,0.350,0.120) \\
B_{7}=(0,0.105,0.155,0.470,0.180)
\end{gathered}
$$

\section{F. Determine the Comprehensive Score}

According to the weighted average method formula, comprehensive score 7 set of efficient solutions can be obtained:

3.0059, 2.9455, 3.1416, 3.6713, 3.7568, 3.5330, 3.7967

From the above score results, that the7th solutions is the highest score, followed by the 5th solutions; the lowest score is the 2 nd solution, followed by 1st solutions can be seen.

\section{CONCLUSION}

Experiment design, artificial neural network, genetic algorithm and fuzzy technology are combined in this paper and a model for solving the multi-objective optimization is constructed based on experimental data. A black box multiobjective optimization problem can be solved effectively 。

It is contradictory that each target is superior in multiobjective optimization, so it is difficult to evaluate multiobjective Pareto optimal solution objectively. Introducing the fuzzy evaluation of multi-objective Pareto optimal solutions is an objective, fair and multi-dimensional evaluation method.

A fuzzy nearness method is applied to evaluate multiobjective Pareto optimal solutions, and fuzzy nearness method is combined with fuzzy comprehensive evaluation, to evaluate multi-objective Pareto optimal solutions with subjective and objective fuzzy comprehensive in the paper. The calculation example shows that this method is effective, and a relatively perfect black box multi-objective optimization system can be formed.

\section{ACKNOWLEDGMENT}

This research was financially supported by the fund of basic scientific research for Chinese center university (N120403008).

\section{REFERENCES}

[1] Horn J, Nafpliotis N, Goldberg D E. A niched Pareto genetic algorithm for multiobjective optimization, Proceedings of the First IEEE Conference on Evolutionary Computation, IEEE World Congress on Computational Intelligence. 1994, 1: pp. 82-87.

[2] Lu H M, Yen Gary G. Rank density based multiobjective genetic algorithm and benchmark test function study, Proceedings of the 2002 Congress on Evolutionary Computation. 2003, 1: pp. 325-343.

[3] Sareni B, Krah enbu hl L, Ni colas A. Niching genetic algorithms for optimization in electromagnetics. I. fundamentals, IEEE Transactions on Magnetics, 1998, 34(5) : pp. 2984-2987 